\title{
ATMOSPHERIC RADIATION BOUNDARY CONDITIONS FOR THE HELMHOLTZ EQUATION
}

\author{
Hélène BarucQ ${ }^{1,2}$, Juliette Chabassier ${ }^{1,2}$, Marc Duruflé ${ }^{1,3}$, Laurent $^{2}$ \\ GizON $^{5,6,4}$ AND Michael LeguÈBE ${ }^{5,6}$
}

\begin{abstract}
This work offers some contributions to the numerical study of acoustic waves propagating in the Sun and its atmosphere. The main goal is to provide boundary conditions for outgoing waves in the solar atmosphere where it is assumed that the sound speed is constant and the density decays exponentially with radius. Outgoing waves are governed by a Dirichlet-to-Neumann map which is obtained from the factorization of the Helmholtz equation expressed in spherical coordinates. For the purpose of extending the outgoing wave equation to axisymmetric or 3D cases, different approximations are implemented by using the frequency and/or the angle of incidence as parameters of interest. This results in boundary conditions called Atmospheric Radiation Boundary Conditions (ARBC) which are tested in ideal and realistic configurations. These ARBCs deliver accurate results and reduce the computational burden by a factor of two in helioseismology applications.
\end{abstract}

Résumé. Ce travail apporte quelques contributions à l'étude numérique des ondes acoustiques se propageant dans le Soleil et son atmosphère. Il se base sur la caractérisation des ondes sortantes dans l'atmosphère représentée par une vitesse constante et une densité décroissant exponentiellement. Les ondes sortantes sont régies par un opérateur Dirichlet-to-Neumann qui est obtenu par la factorisation de l'équation de Helmholtz formulée dans les coordonnées sphériques. Afin d'étendre l'équation des ondes sortantes à des géométries axisymétriques ou 3D, différentes approximations sont menées en utilisant la fréquence et/ou l'angle d'incidence comme paramètres d'intérêt. Ceci mène à des conditions de frontière que nous appelons Conditions de Radiation Atmosphériques (ARBC) et qui sont testées en configuration idéalisées et réalistes. Ces conditions ARBC offrent des résultats précis et réduisent le coût de calcul d'un facteur deux pour le cas du Soleil.

1991 Mathematics Subject Classification. 00A71, 35L05, 85A20, 33C55, 65M60.

December 1, 2017.

Keywords and phrases: Radiation boundary condition, Helmholtz equation, Atmosphere

${ }^{1}$ Magique 3D team, Inria Bordeaux Sud Ouest, 200 avenue de la vieille tour, 33405 Talence Cedex, France

2 LMA, UMR 5142, Université de Pau et des pays de l'Adour, 64000 Pau, France

${ }^{3}$ IMB - Institut de Mathématiques de Bordeaux, 351 cours de la Libération, 33405 Talence, France

${ }^{4}$ Center for Space Science, NYUAD Institute, New York University Abu Dhabi, PO Box 129188, Abu Dhabi, UAE

${ }^{5}$ Max Planck Institut für SonnensystemForschung, Justus-von-Liebig-Weg 3, 37077 Göttingen, Germany

${ }^{6}$ Institut für Astrophysik, Georg-August-Universität Göttingen, Friedrich-Hund-Platz 1, 37077 Göttingen, Germany

(C) EDP Sciences, SMAI 1999 


\section{INTRODUCTION}

Helioseismology is a discipline of solar physics that began to emerge fifty years ago. It uses the study of acoustic waves that propagate inside the Sun in order to infer both its structure and internal dynamics. Oscillations due to Solar acoustic waves can be measured at the Solar surface using a Doppler technique [11]. The original vectorial problem associated with this experimental data can be reduced to an Helmholtz equation with variable coefficients involving the sound speed and the density (see [6], [9]). The problem is set in an infinite domain including the Sun and its atmosphere, and the source term is compactly supported outside the ideal atmosphere. Relevant numerical simulations can then be performed in a bounded domain defined by introducing a boundary bordering the Sun, making possible the use of advanced numerical methods like finite element schemes. The main difficulty is then to find the best boundary condition that is capable of canceling the effect of the artificial external boundary on the acoustic waves. The issue is to address a way of representing outgoing waves into the solar atmosphere by a boundary operator. There have been many studies in the past dealing with the construction of Radiation Boundary Conditions (RBC) for Helmholtz problems (see $[2,3,5]$ ). These conditions have been developed assuming that the medium outside the computational domain is homogeneous. However, in the case of the Sun, the coefficients are not homogeneous, since its atmosphere can be characterized by a density parameter which is exponentially decaying (see [15]). The objective of this paper is to reconsider some techniques formerly used for designing RBCs in the case of constant coefficients and to apply them for deriving boundary conditions to truncate the solar atmosphere. The paper is organized as follows. We begin with a general setting of the equations we are dealing with. By exploiting the radial dependency of the Sun geometry, we propose an approximate factorization of the Helmholtz operator in spherical coordinates. As a result, approximate Dirichlet-to-Neumann conditions are obtained that we propose to call Atmospheric Radiation Boundary Conditions (ARBC). However, these conditions have been obtained assuming that the Sun is a perfect sphere. In the case of other shapes, by performing the microlocal factorization of the Helmholtz problem proposed in [2], we obtain Non Spherical Atmospheric Radiation Boundary Conditions extending the scope of application of ARBCs to a large variety of application domains. Numerical results are then performed for a sphere and an ellipsoid and are completed by realistic numerical tests into the atmosphere of the Sun.

\section{General Setting of the Problem}

The acoustic waves propagating in the Sun and its atmosphere can be represented as the solution $u$ to the Helmholtz equation [9]:

$$
-\frac{\omega^{2}}{\rho c^{2}} u-\operatorname{div}\left(\frac{1}{\rho} \nabla u\right)=f
$$

set in the whole space $\mathbb{R}^{3}$. The infinite propagation domain is characterized by a density function $\rho$ and a sound speed (also called celerity or velocity) $c$ that are varying along with the radial variable $r=|\mathbf{x}|, \mathbf{x} \in \mathbb{R}$ :

$$
\rho(\mathbf{x}) \equiv \rho(r), \quad c(\mathbf{x}) \equiv c(r) .
$$

In the following, we shall distinguish two domains. The first one is a bounded sphere of radius $R_{a}$ in which the parameters $\rho$ and $c$ are given by the Model S of [8]. The second one is its complement in $\mathbb{R}^{3}$, in which the parameters $\rho$ and $c$ are supposed to follow an ideal atmospherical behavior prompted by [15]: $c$ is constant and $\rho$ is exponentially decaying. We will respectively call these domains the "interior" of the Sun $\left\{\mathbf{x} ;|\mathbf{x}| \leq R_{a}\right\}$ and "atmosphere" of the Sun $\left\{\mathbf{x} ;|\mathbf{x}|>R_{a}\right\}$. The atmosphere is therefore in this paper defined as the geometrical region in which the coefficients follow the ideal atmospherical behavior ${ }^{1}$. More precisely, following [15], we assume that there

\footnotetext{
${ }^{1}$ Notice that, since the radius $R_{a}$ is strictly greater than the "surface" of the Sun $R_{\odot}$, these definitions are not consistent with the usual definition for the Sun's interior : $\left\{\mathbf{x} ;|\mathbf{x}| \leq R_{\odot}\right\}$ but will be used in this paper, where we are only interested in the atmosphere modeling, for the sake of simplicity.
} 
exists a radius $R_{a}$ and a positive constant $\alpha$ such that:

$$
\rho(r)=\left\{\begin{array}{ll}
\rho^{-}(r) \geq \rho_{0}>0 & \text { if } r \leq R_{a} \\
\rho^{-}\left(R_{a}\right) e^{-\alpha\left(r-R_{a}\right)} & \text { if } r>R_{a}
\end{array}, \quad c(r)=\left\{\begin{array}{ll}
c^{-}(r) \geq c_{0}>0 & \text { if } r \leq R_{a} \\
c^{-}\left(R_{a}\right)=c_{a} & \text { if } r>R_{a}
\end{array} .\right.\right.
$$

We will moreover suppose that the support of the source term $f$ is included in the Sun's interior. The pulsation $\omega$ is complex and incorporates a damping parameter $\gamma$ :

$$
\omega=\left(1+\frac{2 i \gamma}{\omega_{0}}\right)^{1 / 2} \omega_{0}
$$

The real pulsation $\omega_{0}$ is given by $\omega_{0}=2 \pi f_{0}$, where $f_{0}$ is the frequency. The definition of the complex square root (denoted ${ }^{1 / 2}$ ) is detailed in the next section. The damping parameter $\gamma$ is assumed to be a strictly positive constant such that the Helmholtz equation can be completed by a Dirichlet condition

$$
\lim _{|\mathbf{x}| \rightarrow+\infty} u=0 .
$$

The radial dependency of the geometry prompts us to seek the solution $u$ under the form

$$
u(r, \theta, \phi)=\sum_{\ell=0}^{L} \sum_{m=-\ell}^{\ell} u^{\ell, m}(r) Y_{\ell}^{m}(\theta, \phi),
$$

where $Y_{\ell}^{m}$ are the spherical harmonics, given by

$$
Y_{\ell}^{m}(\theta, \phi)=(-1)^{m} \sqrt{\frac{(2 \ell+1)}{4 \pi} \frac{(\ell-m) !}{(\ell+m) !}} P_{\ell}^{m}(\cos \theta) e^{i m \phi} .
$$

$P_{\ell}^{m}$ are the associated Legendre polynomials, their maximal degree used in the expression of $u$ being denoted by $L$. The equation satisfied by $u^{\ell, m}$ is

$$
-\frac{\omega^{2} r^{2}}{\rho c^{2}} u^{\ell, m}-\frac{\partial}{\partial r}\left(\frac{r^{2}}{\rho} \frac{\partial u^{\ell, m}}{\partial r}\right)+\frac{\ell(\ell+1)}{\rho} u^{\ell, m}=r^{2} f^{\ell, m},
$$

where

$$
f^{\ell, m}=\int_{0}^{\pi} \int_{0}^{2 \pi} f(r, \theta, \phi) \overline{Y_{\ell}^{m}}(\theta, \phi) \sin \theta d \phi d \theta .
$$

Supposing that the density $\rho$ is smooth enough, we can consider the equivalent equation:

$$
-\frac{\omega^{2}}{c^{2}} u^{\ell, m}-\frac{1}{r^{2}} \frac{\partial}{\partial r}\left(r^{2} \frac{\partial u^{\ell, m}}{\partial r}\right)+\frac{\ell(\ell+1)}{r^{2}} u^{\ell, m}-\alpha \frac{\partial u^{\ell, m}}{\partial r}=\rho f^{\ell, m},
$$

where the coefficient $\alpha$ is given by :

$$
\alpha(r)=-\frac{\rho^{\prime}(r)}{\rho(r)}
$$

and where $\rho^{\prime}$ denotes the derivative of $\rho$ with respect to $r$. It is worth noting that in the atmosphere $\left(r>R_{a}\right), \alpha$ is a positive constant.

Whereas the propagation domain is infinite, the domain of interest in which numerical simulations are relevant can be reduced to a bounded domain limited by an external boundary surrounding the Sun interior. The accuracy of the numerical solution depends then on the boundary condition that is applied on the external boundary. In the following, we focus on the construction of such boundary conditions once the problem has been reduced to Eq. (8) with constant sound speed, and variations of density taken into account through the parameter $\alpha$. 


\section{DERIVATION OF RADiATion BOUndARY CONDITIONS}

As previously introduced, we aim at deriving efficient boundary conditions to truncate the computational domain. In the following, we propose to derive them by writing the variations of the acoustic waves along the normal direction, which amounts to represent the Dirichlet-toNeumann operator associated with the problem, and to select waves that are outgoing to a given surface.

\subsection{Outgoing radiating waves}

We consider the homogeneous equation set in the atmosphere $\left(r \geq R_{a}\right)$ :

$$
-\frac{\omega^{2}}{c^{2}} v-\frac{1}{r^{2}} \frac{\partial}{\partial r}\left(r^{2} \frac{\partial v}{\partial r}\right)+\frac{\ell(\ell+1)}{r^{2}} v-\alpha \frac{\partial v}{\partial r}=0 .
$$

We propose to characterize outgoing radiating waves which satisfy the previous equation by factorizing of the problem. This can be done by observing that:

$$
\begin{aligned}
\frac{1}{r^{2}} \frac{\partial}{\partial r}\left(r^{2} \frac{\partial v}{\partial r}\right)+\alpha \frac{\partial v}{\partial r} & =\frac{\partial^{2} v}{\partial r^{2}}+\left(\frac{2}{r}+\alpha\right) \frac{\partial v}{\partial r} \\
& =\left(\frac{\partial}{\partial r}+\left(\frac{1}{r}+\frac{\alpha}{2}\right)\right)^{2} v-\frac{\partial}{\partial r}\left(\frac{1}{r}+\frac{\alpha}{2}\right) v-\left(\frac{1}{r}+\frac{\alpha}{2}\right)^{2} v
\end{aligned}
$$

Since $\alpha$ is assumed to be constant in the atmosphere, we get

$$
\left(\frac{\partial}{\partial r}+\left(\frac{1}{r}+\frac{\alpha}{2}\right)\right)^{2} v=-\frac{1}{c^{2}}\left(\omega^{2}-\omega_{c, \ell}^{2}\right) v
$$

where we introduced the real quantity $\omega_{c, \ell}(r)$ defined by:

$$
\frac{\omega_{c, \ell}^{2}(r)}{c^{2}}=-\frac{1}{r^{2}}+\left(\frac{1}{r}+\frac{\alpha}{2}\right)^{2}+\frac{\ell(\ell+1)}{r^{2}} .
$$

In the following, $\omega_{c, \ell} /(2 \pi)$ is called the cut-off frequency. If $\omega_{0}$ is smaller (resp. greater) than $\omega_{c, \ell}(r)$ (and not too close), the leading behavior of the solution of (11) is evanescent (resp. propagating). There is no clear leading behavior when $\omega_{0}$ is close to $\omega_{c, \ell}$. To compute the square-root of $\frac{1}{c^{2}}\left(\omega^{2}-\omega_{c, \ell}^{2}\right)$, we use the principal branch of the function $z^{1 / 2}$ defined on the complex plane $\mathbb{C}-\mathbb{R}^{-}$by $z^{1 / 2}=\sqrt{|z|} \exp (i \theta / 2)$ for $\theta$ in $]-\pi, \pi\left[\right.$. Then the real part of $\left(\omega^{2}-\omega_{c, \ell}^{2}\right)^{1 / 2}$ never vanishes because $\omega$ has a non-zero imaginary part. Thus, according to [14], Eq. (11) governs propagating waves which are outgoing or incoming, depending on the sign of the real part of $\left(\omega^{2}-\omega_{c, \ell}^{2}\right)^{1 / 2}$. Then outgoing waves $v^{+}$, that we choose propagating in the direction of increasing $r$, are given as the solutions to:

$$
\left(\frac{\partial}{\partial r}+\left(\frac{1}{r}+\frac{\alpha}{2}\right)\right) v^{+}=i\left(\frac{\omega^{2}}{c^{2}}-\frac{\omega_{c, \ell}^{2}(r)}{c^{2}}\right)^{1 / 2} v^{+},
$$

for a fixed value of $r$ defining the surface from which waves are outgoing. Similarly, we can characterize incoming waves $v^{-}$as the solutions to

$$
\left(\frac{\partial}{\partial r}+\left(\frac{1}{r}+\frac{\alpha}{2}\right)\right) v^{-}=-i\left(\frac{\omega^{2}}{c^{2}}-\frac{\omega_{c, \ell}^{2}(r)}{c^{2}}\right)^{1 / 2} v^{-} .
$$


Remark. Although we have characterized outgoing and incoming waves, we cannot say that the initial equation governing (9) can be written as the product of the two corresponding equations. Indeed, if we compute the commutator between the outgoing and incoming operators, we get $i \frac{\partial}{\partial r}\left(\left(\frac{\omega^{2}}{c^{2}}-\frac{\omega_{c, \ell}^{2}(r)}{c^{2}}\right)^{1 / 2}\right)$. Given the definition of the cut-off frequency, we see that this commutator depends on the variations of the velocity and also on the distance of truncation. In fact, the velocity is considered constant into the atmosphere. The distance of truncation is thus the parameter of interest and we observe that the value of the commutator decays to zero as the parameter $r$ increases. We are thus retrieving a very well-known property: the further the external boundary, the more precise the outgoing condition. Anyway, the expression of the commutator shows that outgoing waves are represented by Eq. (13) modulo a regular function.

In the following, we thus propose to apply condition (13) on a surface surrounding the Sun and located at a given radius $r=R$. We denote by $\kappa=1 / R$ the mean curvature of the sphere with radius $R$. An outgoing radiation condition for solutions to Eq. (1) may then be given by:

$$
\frac{\partial u}{\partial r}=-\left(\kappa+\frac{\alpha}{2}\right) u+\frac{i \omega}{c}\left(1-\frac{c^{2}}{\omega^{2}}\left(\frac{\ell(\ell+1)}{R^{2}}+\kappa \alpha+\frac{\alpha^{2}}{4}\right)\right)^{1 / 2} u . \quad \text { (Atmo RBC Non Local) }
$$

It is worth noting that this condition depends on the spherical harmonics degree $\ell$ (mode number) and involves a square-root. The term involving $\ell$ corresponds exactly, in the context where the radial formulation is not possible, to the symbol of the Laplace Beltrami operator, which square root is a nonlocal operator. In practice, this leads to extra computational costs and it is the reason why in the following of the paper, we will propose some approximations of this condition to overcome this problem.

Remark. As previously observed, the imaginary part of $\omega$ never vanishes, which implies that the frequency regime always corresponds mathematically to propagating waves. Nevertheless, when the imaginary part of $\left(\omega^{2}-\omega_{c, \ell}^{2}\right)^{1 / 2}$ is large enough with respect to the real part, the outgoing wave is close to an evanescent wave because the characteristic time of exponential decay is smaller than the characteristic time of oscillation. This phenomenon appears when

$$
\omega_{0} \leq \min \left(\frac{\omega_{c, \ell}^{2}}{2 \gamma}, \omega_{c, \ell}\right)
$$

This means that in the low-frequency regime, the condition (Atmo $\mathrm{RBC}$ Non Local) might be not well-suited and asymptotic conditions obtained as in [7] should be more relevant.

\subsection{Mathematical study of the RBC for the solar atmosphere}

To give a sense to the RBC we have proposed, we prove that the problem set into a truncated domain and governed by the Helmholtz equation coupled with the RBC is well-posed. The problem can be set into the Sobolev space $H^{2}(\Omega)$ where $\Omega$ denotes the bounded region limited by the circular boundary $r=R$. It turns out that the problem is of Fredholm type, observing that it can be written as a compact perturbation of the identity. This is a standard result because the radiation boundary condition can be rewritten as an impedance condition as follows:

$$
\frac{\partial}{\partial \mathbf{n}} u=\mathbf{Z} u
$$


with $\mathbf{Z}$ defined by:

$$
\mathbf{Z}=-\left(\kappa+\frac{\alpha}{2}\right)+\frac{i \omega}{c} \sqrt{1-\frac{c^{2}}{\omega^{2}}\left(\frac{\ell(\ell+1)}{R^{2}}+\kappa \alpha+\frac{\alpha^{2}}{4}\right)} .
$$

The imaginary part of the impedance operator is, by construction, positive. It is a sufficient condition to get uniqueness for the mixed problem which also implies existence of a solution.

\subsection{Approximate Atmospheric Radiation Boundary Conditions}

The previous Radiation Boundary Condition (Atmo RBC Non Local) that has been deduced from the factorization procedure can be implemented easily in a radial configuration but has a major drawback : it leads to a spatially non local operator both in axisymmetric or 3D geometries. To overcome this classical issue, we propose approximate Atmospheric Radiation Boundary Conditions, obtained by performing asymptotic expansions in different regimes. Let $R \geq R_{a}$ be as before the radius of the artificial boundary on which we want to derive our radiation conditions. We still call $\kappa=1 / R$ the mean curvature of the sphere.

A first way to localize the condition is to expand the square root for high frequencies, this gives the following conditions which neglects terms in $\mathcal{O}(1 / \omega)$

$$
\frac{\partial u}{\partial r}=-\left(\kappa+\frac{\alpha}{2}\right) u+\frac{i \omega}{c} u
$$

(Atmo RBC HF 0)

Remark. If $\alpha=0$ (constant density), this condition is identical to the condition of order 1 found for homogeneous media in Eq. (21) of [2]. As we will show later on, the procedure followed in [2] can be generalized to the case where the density is not constant.

If we neglect terms in $\mathcal{O}\left(1 / \omega^{2}\right)$, we get

$$
\frac{\partial u}{\partial r}=-\left(\kappa+\frac{\alpha}{2}\right) u+\frac{i \omega}{c} u+\frac{c}{2 i \omega}\left(\frac{\ell(\ell+1)}{R^{2}}+\kappa \alpha+\frac{\alpha^{2}}{4}\right) u
$$

(Atmo RBC HF 1)

Remark. If $\alpha=0$ (constant density), this condition is similar to the condition of order $3 / 2$ found for homogeneous media in Eq. (22) of [2], since the term $\frac{\ell(\ell+1)}{R^{2}}$ is the radial expression for the Laplace-Beltrami operator $\Delta_{\Gamma}$.

Other asymptotic formulae can be chosen in order to expand the square root, as for instance the "small angle of incidence" point of view. The small parameter is not considered to be the inverse of the pulsation $1 / \omega$, but the quantity $\ell(\ell+1) /\left(R^{2} \omega^{2}\right)$, which is monotonic with the local angle of incidence with respect to the normal of the spherical boundary. In this configuration, we obtain the following condition by retaining terms in $\mathcal{O}\left(\ell(\ell+1) /\left(R^{2} \omega^{2}\right)\right)$

$$
\frac{\partial u}{\partial r}=-\left(\kappa+\frac{\alpha}{2}\right) u+\frac{i \omega}{c} \sqrt{1-\frac{c^{2}}{\omega^{2}}\left(\kappa \alpha+\frac{\alpha^{2}}{4}\right)} u+\frac{\frac{c}{2 i \omega} \frac{\ell(\ell+1)}{R^{2}}}{\sqrt{1-\frac{c^{2}}{\omega^{2}}\left(\kappa \alpha+\frac{\alpha^{2}}{4}\right)}} u \text {. (Atmo SAI 1) }
$$


A final radiation boundary condition is given by neglecting in the above expression the terms involving the curvature and the tangential derivative, which gives the very simple condition

$$
\frac{\partial u}{\partial r}=-\frac{\alpha}{2} u+\frac{i \omega}{c} \sqrt{1-\frac{c^{2} \alpha^{2}}{4 \omega^{2}}} u
$$

(Atmo RBC 1)

\subsection{Radiation conditions for non spherical geometries}

In this paragraph, we aim at deriving conditions that can be used in a more general framework where the solution is not necessarily radial. Let $\Gamma$ be the smooth enough surface of $\mathbb{R}^{3}$ on which the condition is set. Following [2], we introduce a local coordinate system $\left(r, s_{1}, s_{2}\right)$ that corresponds to the principal basis of the tangent plane of boundary $\Gamma$. Eq. (1) can then be written as

$$
\partial_{r}^{2} u+\left(2 \mathcal{H}_{r}+\alpha\right) \partial_{r} u+\frac{1}{h_{1} h_{2}}\left(\partial_{s_{1}}\left(\frac{h_{2}}{h_{1}} \partial_{s_{1}} u\right)+\partial_{s_{2}}\left(\frac{h_{1}}{h_{2}} \partial_{s_{2}} u\right)\right)+\frac{\omega^{2}}{c^{2}} u=0
$$

where $\mathcal{H}_{r}$ is the mean curvature of the parallel surface $\Gamma_{r}$. It is defined by

$$
\mathcal{H}_{r}=\frac{1}{2} \frac{\partial_{r}\left(h_{1} h_{2}\right)}{h_{1} h_{2}}
$$

where $h_{i}(r)=1+r \mathcal{C}_{i}, \mathcal{C}_{1}$ and $\mathcal{C}_{2}$ being the principal curvatures of $\Gamma$. We denote $\mathcal{G}=\mathcal{C}_{1} \mathcal{C}_{2}$ the Gauss curvature and $\mathcal{H}=\left(\mathcal{C}_{1}+\mathcal{C}_{2}\right) / 2$ the mean curvature of $\Gamma$. Note that $\mathcal{C}_{1}=\mathcal{C}_{2}=1 / R$ in the case of a spherical geometry. This equation has the same structure as Eq. (7) of [2], and the same techniques can be applied to obtain the following second-order Atmospheric Radiation Boundary Condition

$$
\begin{aligned}
& \frac{\partial u}{\partial r}-\frac{i \omega}{c} u+(\mathcal{H}+\left.\frac{\alpha}{2}\right) u-\frac{c}{2 i \omega}\left[\left(\mathcal{G}-\mathcal{H}^{2}\right)\left(1+\frac{2 c}{i \omega} \mathcal{H}\right)+\alpha \mathcal{H}+\frac{\alpha^{2}}{4}+\frac{\alpha^{\prime}}{4}\right. \\
&\left.+\frac{c}{i \omega}\left(\frac{\alpha \mathcal{H}+\alpha^{\prime} \mathcal{G}}{2}-\alpha \mathcal{H}^{2}+\frac{\alpha^{\prime} \alpha+\alpha^{\prime \prime}}{4}\right)\right] u-\frac{\Delta_{\Gamma} \mathcal{H} c^{2}}{4 \omega^{2}} u \\
& \quad+\operatorname{div}_{\Gamma}\left(\frac{c}{2 i \omega}\left(\mathcal{I}-\frac{c i \mathcal{R}}{\omega}\right) \nabla_{\Gamma}\right) u=0 .
\end{aligned}
$$

In the radial configuration, where $\mathcal{H}=\kappa$ and $\mathcal{G}=\kappa^{2}$, since $\alpha$ is constant in the atmosphere, the previous condition becomes:

$$
\begin{aligned}
\frac{\partial u}{\partial r}=-\left(\kappa+\frac{\alpha}{2}\right) u+\frac{i \omega}{c} u+\frac{c \alpha}{2 i \omega} & \left(\kappa+\frac{\alpha}{4}+\frac{c \kappa}{2 i \omega}(1-2 \kappa)\right) u \\
& +\frac{c}{2 i \omega}\left(1+\frac{c \kappa}{i \omega}\right) \frac{\ell(\ell+1)}{R^{2}} u . \quad \text { (Atmo RBC Non Spherical) }
\end{aligned}
$$

It is worth noting that conditions (20) and (Atmo RBC Non Spherical) are valid only for highfrequency waves. 
Remark. The condition of order $3 / 2$ (obtained by a high frequency truncation of order 1 instead of 2 of the symbols of the operators) reads

$$
\frac{\partial u}{\partial r}-\frac{i c}{\omega} u+\left(\mathcal{H}+\frac{\alpha}{2}\right) u+\frac{c}{2 i \omega} \Delta_{\Gamma} u-\frac{c}{2 i \omega}\left[\left(\mathcal{H}+\frac{\alpha}{2}\right)^{2}+\mathcal{G}-2 \mathcal{H}^{2}+\frac{\alpha^{\prime}}{2}\right] u=0,
$$

which, in the case of a spherical geometry, is very similar to (Atmo RBC HF 1) (up to $\frac{c}{2 i \omega} \mathcal{H}^{2} u$ ).

\section{Numerical method AND ASSESSMENT OF THE CONDitions}

All the numerical results have been obtained using the Montjoie ${ }^{2}$ finite elements library (see [6] for details about the numerical method).

We denote by $R \geq R_{a}$ the maximal radius of the computational domain, where the different boundary conditions will be set. The interval $[0, R]$ is divided into subintervals:

$$
[0, R]=\bigcup\left[x_{i}, x_{i+1}\right]
$$

One dimensional finite elements will be used in $r$-coordinate. The 1D finite element space is given by

$$
V_{h}=\left\{u \in H^{1}([0, R]) \text { such that }\left.u\right|_{\left[x_{i}, x_{i+1}\right]} \in \mathbb{P}_{p}\right\} .
$$

where $\mathbb{P}_{p}$ is the space of polynomials of degree lower or equal to $p, p$ being the order of the approximation. The solution $u^{\ell, m}$ is then searched under the form

$$
u^{\ell, m}(r)=\sum_{i=0}^{N_{h}} u_{i}^{\ell, m} \varphi_{i}(r) .
$$

where $\varphi_{i}$ are basis functions generating the finite element space $V_{h}$ of dimension $N_{h}$. The variational formulation solved by $u^{\ell, m}$ is obtained from (6) and reads

$$
\begin{aligned}
-\omega^{2} \int_{0}^{R} \frac{1}{\rho c^{2}} r^{2} u^{\ell, m} \varphi_{i} d r+\int_{0}^{R} \frac{r^{2}}{\rho} \frac{\partial u^{\ell, m}}{\partial r} \frac{\partial \varphi_{i}}{\partial r} d r+ & \\
& \ell(\ell+1) \int_{0}^{R} \frac{1}{\rho} u^{\ell, m} \varphi_{i} d r-\left[\frac{1}{\rho} r^{2} \frac{\partial u^{\ell, m}}{\partial r} \varphi_{i}\right]_{0}^{R}=f_{i}^{\ell, m},
\end{aligned}
$$

where

$$
f_{i}^{\ell, m}=\int_{0}^{R} \int_{0}^{\pi} \int_{0}^{2 \pi} r^{2} f(r, \theta, \phi) \overline{Y_{\ell}^{m}}(\theta, \phi) \sin \theta d \phi d \theta \varphi_{i} d r
$$

The boundary term in square brackets of the variational formulation is replaced by the correct term depending on the boundary condition imposed at $r=R$. A homogeneous Neumann $\mathrm{BC}$ is imposed at $r=0$. Gauss-Lobatto points are used both as interpolation and quadrature points. The source term is computed using Gauss-Legendre integration formulae.

In radial configuration, the proposed Atmospheric Boundary Conditions all take the form

$$
\frac{\partial u^{\ell, m}}{\partial r}(R)=Z^{\ell}(R) u^{\ell, m}(R)
$$

where the value of the impedance coefficient $Z^{\ell}(R)$ differs from one condition to the other. The numerical burden is therefore the same for all conditions applied on the same mesh.

\footnotetext{
${ }^{2}$ montjoie.gforge.inria.fr
} 


\subsection{Exact boundary condition and computation of a reference solution}

This section is devoted to the description of a transparent boundary condition, which will be used to compute reference solutions, in order to assess the precision of the different RBCs. Inside the atmosphere, the following differential equation holds:

$$
r^{2}\left(u^{\ell}\right)^{\prime \prime}+2 r\left(u^{\ell}\right)^{\prime}+\alpha r^{2}\left(u^{\ell}\right)^{\prime}+\left[k_{\infty}^{2} r^{2}-\ell(\ell+1)\right] u^{\ell}=0,
$$

where

$$
k_{\infty}=\frac{\omega}{c_{a}} .
$$

If $\alpha=0$, the elementary solutions are the spherical Hankel functions $h_{\ell}^{(1)}(r)$ and $h_{\ell}^{(2)}(r)$, an exact condition is then given as :

$$
\frac{\partial u^{\ell}}{\partial r}=\frac{k_{\infty} h_{\ell}^{(1)^{\prime}}\left(k_{\infty} R\right)}{h_{\ell}^{(1)}\left(k_{\infty} R\right)} u^{\ell} .
$$

A way to obtain an exact condition for $\alpha \neq 0$ would be to compute exact solutions of the differential equation Eq. (27), and deduce the Dirichlet-to-Neumann operator. Here, we have chosen to compute numerically this Dirichlet-to-Neumann operator by computing the solutions $w^{\ell}$ to the exterior problem :

$$
\left\{\begin{array}{l}
r^{2}\left(w^{\ell}\right)^{\prime \prime}+2 r\left(w^{\ell}\right)^{\prime}+\alpha r^{2}\left(w^{\ell}\right)^{\prime}+\left[k_{\infty}^{2} r^{2}-\ell(\ell+1)\right] w^{\ell}=0 \text { for } r \geq R, \\
w^{\ell}(R)=1 .
\end{array}\right.
$$

We use the value of $\frac{\partial w^{\ell}}{\partial r}(R)$ as the exact impedance $Z_{\text {exact }}^{\ell}(R)$, and we impose as BC

$$
\frac{\partial u^{\ell}}{\partial r}(R)=Z_{\text {exact }}^{\ell}(R) u^{\ell}(R)
$$

when solving the physical problem in $[0, R]$. When solving Eq. (27), we remove the phase by representing $u$ as

$$
u^{\ell}=v \exp (\beta r), \quad \text { with } \beta=-\frac{\alpha}{2}+i \sqrt{k_{\infty}^{2}-\frac{\ell(\ell+1)}{R^{2}}-\frac{\alpha^{2}}{4}} .
$$

As a result, $v$ will not decrease too rapidly or vary too rapidly and can be discretized with highorder finite elements. Removing this phase prevents the solution from going below $10^{-300}$, which ensures that double precision is accurate enough.

\subsection{Toy problem}

As a toy problem to assess the different conditions, we propose to consider a celestial body of atmosphere radius $R_{a}=1$ with a piecewise exponential density and a constant sound speed and damping. More precisely,

$$
\begin{gathered}
\rho(r)=\left\{\begin{array}{ll}
2 & \text { if } r \leq R_{a} \\
2 e^{-\alpha(r-1)} & \text { if } r>R_{a}
\end{array}, \quad c(r)=c_{0}=3.0, \quad \gamma=\gamma_{0}=10.0,\right. \\
\alpha \in\left\{20,50,5 \times 10^{3}\right\}, \quad f_{0} \in[2.0,128.0] \mathrm{Hz} .
\end{gathered}
$$

The computation of the numerical solution will be done on several computational domains $[0, R]$, where $R$ will be chosen in $\left[R_{a}, 3 R_{a}\right]$. The point $r=R_{a}$ is a vertex of the mesh. If $R=R_{a}$ the condition is imposed directly where the density becomes exponentially decaying, which is a difficult case (On Surface Radiation Condition, [1]). Finite elements of order 16 are used on a regular grid with a space step $\Delta x=0.025$. This space discretization ensures at least 10 degrees 

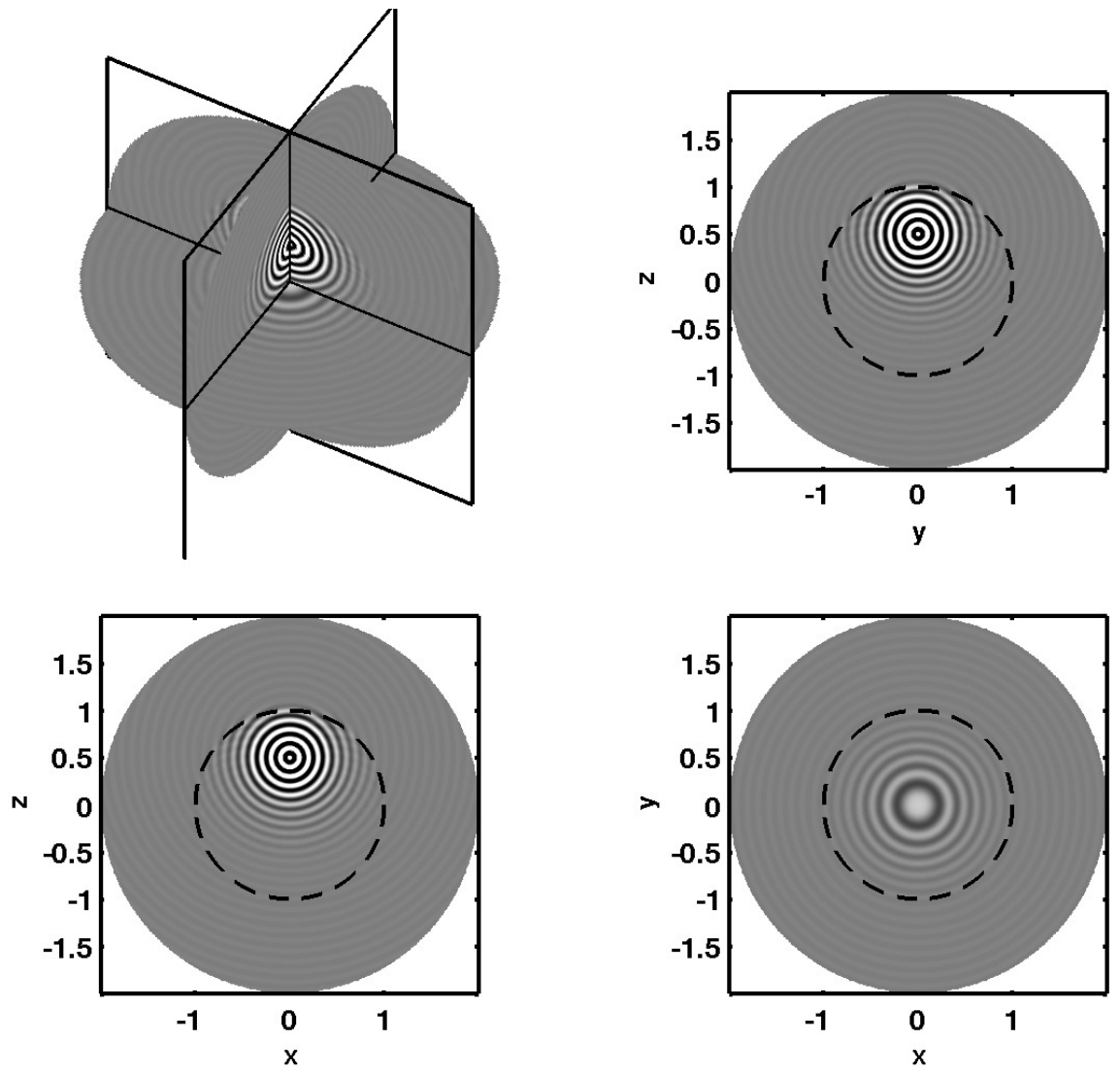

Figure 1. Real part of the exact solution using parameters (28) and (32). The dashed line represents $r=R_{a}$.

of freedom per wavelength for all considered frequencies $f_{0}$. The source is a Gaussian located at $\mathbf{x}_{\mathbf{0}}=(0,0,0.5)$ of radius $r_{0}=0.05$ :

$$
f(\mathbf{x})=e^{-\log \left(10^{-6}\right) \frac{\left\|\mathbf{x}-\mathbf{x}_{\mathbf{0}}\right\|^{2}}{r_{0}^{2}}}
$$

The mesh is refined around the source point $r=0.5$. The number of spherical harmonics $L$ is chosen automatically such that

$$
\left\|u^{\ell, m}\right\|_{\infty} \leq 10^{-14}, \quad \forall \ell>L+1
$$

In practice, in the considered cases, $L$ is not larger than 400 .

\subsubsection{Value of the impedance coefficient}

First, we compare the value of the impedance coefficient $Z^{\ell}(R)$ of the different radiation boundary conditions with $Z_{\text {exact }}^{\ell}(R)$ which is obtained as described above. We expect that a good approximation of this coefficient will later lead to a good numerical solution. The following configuration is considered:

$$
\alpha=50, \quad R=2.0, \quad f_{0}=30.0 \text {. }
$$




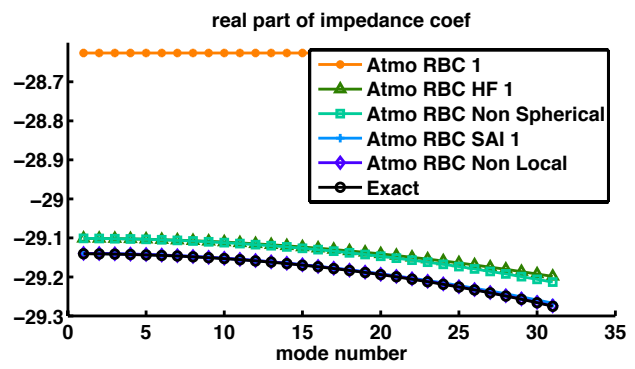

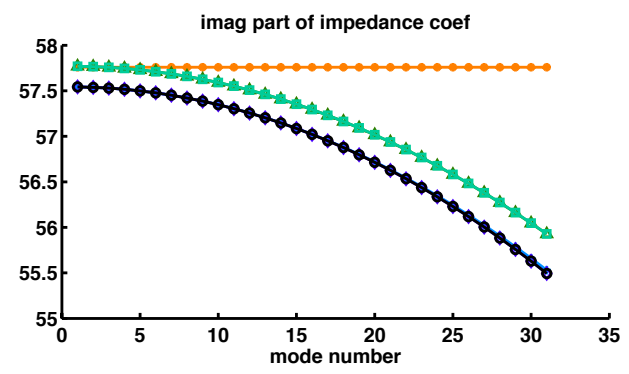

(a) Real and imaginary part

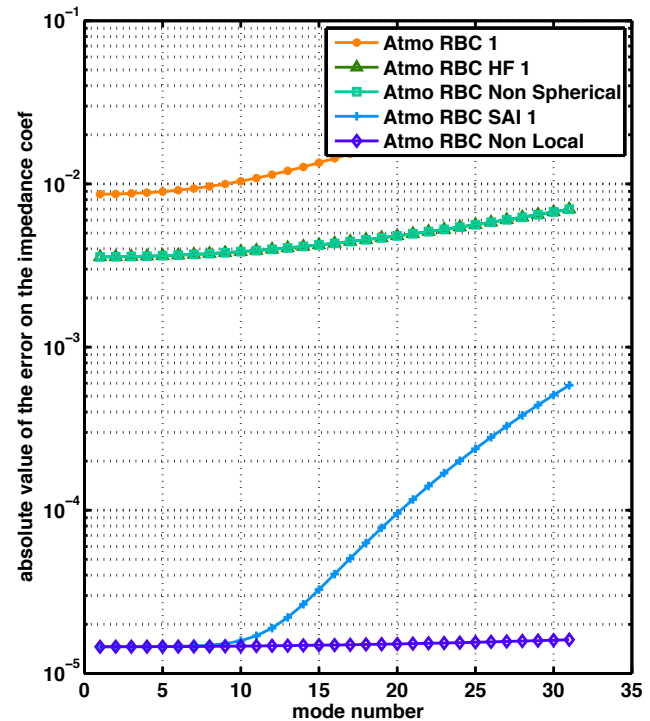

(b) Relative absolute value of error

FIGURE 2. Impedance coefficient obtained with the various radiation boundary conditions.

The numerical solution obtained with the exact impedance coefficient is displayed in Fig. 1. The comparison of approximate and exact impedance coefficients are displayed in Fig. 2 for the 30 first spherical harmonics. The exact impedance is displayed in black. The condition (Atmo RBC 1) provides the worst results, it is the only condition that does not account for the curvature of the boundary. Even if, in this case, $\kappa=0.5$ while $\alpha / 2=25$, this correction appears to be very important. Moreover, this condition does not involve a surface derivative term, which induces a loss of precision as the mode number increases. The second less accurate conditions are (Atmo RBC HF 1) and (Atmo RBC Non Spherical), which almost coincide in the figures. They both come from an expansion of the square root for high frequencies, which explains the discrepancy even for the first mode $(\ell=0)$. However, the tendency as $\ell$ increases is well captured. The condition (Atmo SAI 1) leads to very nice results. The absolute value for low number modes is well approximated, and the tendency as $\ell$ increases is well captured. The only better condition is (Atmo RBC Non Local), which deteriorates very slowly as $\ell$ increases. Unfortunately, the condition cannot be localized for 3D or axisymmetric simulations. The error for this condition is around $1.6 \cdot 10^{-5}$, which quantifies the error made during the factorization of Section 3.1.

\subsubsection{Influence of the distance of the artificial boundary}

In this section and the following, the numerical solution is computed using the different radiation boundary conditions, and compared to the solution obtained using the exact impedance coefficients. Finally, we also compare to the use of homogeneous Dirichlet boundary condition

$$
u=0,
$$

(Dirichlet BC)

and to the naive use of a Sommerfeld-like condition:

$$
\frac{\partial u}{\partial r}-\frac{i \omega}{c_{a}} u=0
$$

(Naive Sommerfeld RBC)

where $i \omega / c_{a}$ is the interior wave number in our case. The error is a relative $L^{2}$ error on the radius $(x, y)=0, z \in\left[0, R_{a}\right]$. The results are displayed in Fig. 3 for a frequency $f_{0}=30.0$ 
and for two values of $\alpha \in\left\{20,5 \cdot 10^{3}\right\}$. We observe that for all conditions, the error decreases as the artificial boundary moves away. In the family of presently derived radiation boundary conditions, (Atmo RBC Non Local) performs best, followed very closely by (Atmo SAI 1). The two conditions obtained after a high frequency expansion, (Atmo RBC Non Spherical) and (Atmo RBC HF 1), perform less well, especially when $\alpha$ is big. Naive conditions (Dirichlet BC) and (Naive Sommerfeld RBC) lead to large error values.

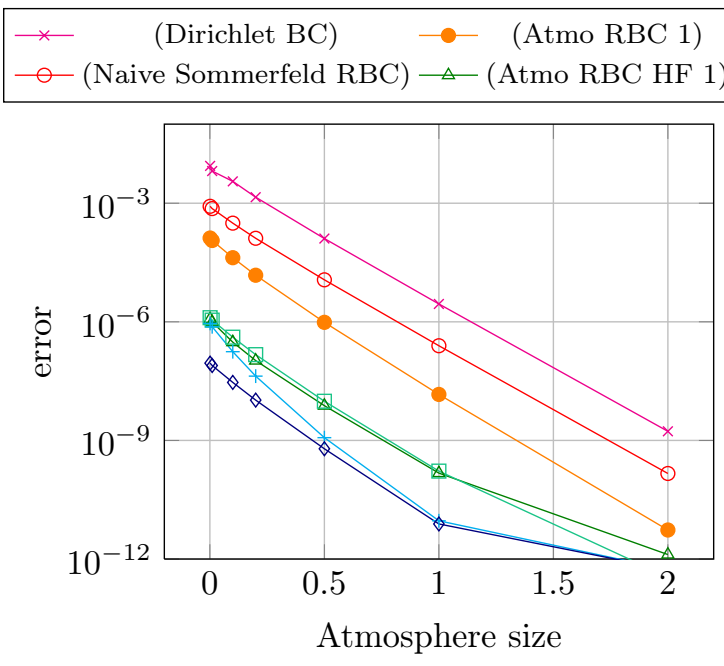

(a) $\alpha=20$ $\square$ (Atmo RBC Non Spherical $) \multimap($ Atmo RBC Non Local $)$ (Atmo SAI 1)

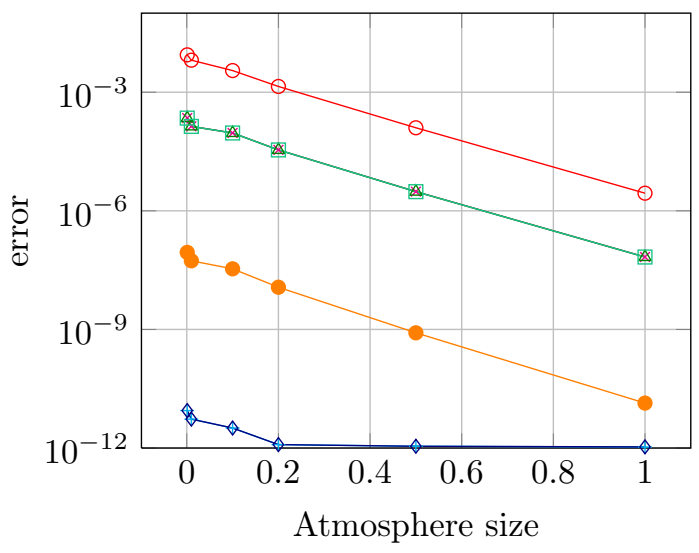

(b) $\alpha=5 \times 10^{3}$

FiguRE 3. $L^{2}$ relative errors with reference solution, with respect to the radius of the artificial boundary. The frequency is set to $f_{0}=30.0 \mathrm{~Hz}$.

\subsubsection{Influence of the atmospheric density decay}

In this section we explore the influence of the parameter $\alpha$ in the same configuration as the previous section. The conditions are imposed directly on the atmosphere radius $R_{a}$ and the frequency takes two values $f_{0} \in\{5.0,30.0\}$. The results are displayed in Fig. 4. All conditions give better results as $\alpha$ gets large, except one: the naive Sommerfeld condition (Naive Sommerfeld RBC), for which the error made on the solution is very large and increases. This illustrates that using the naive Sommerfeld condition is not well suited to this configuration. Once again, the best proposed condition is (Atmo RBC Non Local), followed very closely by (Atmo SAI 1), while (Atmo RBC 1) provides satisfying results.

\subsubsection{Influence of the frequency}

In this last section on the toy problem, we explore the influence of the frequency on the accuracy of the conditions. The conditions are again imposed directly on the atmosphere radius $R_{a}$ and the atmosphere parameter $\alpha$ takes two values $\alpha \in\left\{50,5 \cdot 10^{3}\right\}$. The results are displayed in Fig. 5. Different orders of convergence seem to emerge, in accordance with the fact that some conditions are obtained using a high frequency approximation while some are supposing a small angle of incidence. Once again, the best proposed condition in the whole frequency range is (Atmo RBC Non Local), followed very closely by (Atmo SAI 1), while (Atmo RBC 1) provides satisfying results for low frequencies. For high frequencies, (Atmo RBC Non Spherical) and (Atmo RBC HF 1) perform very well. 


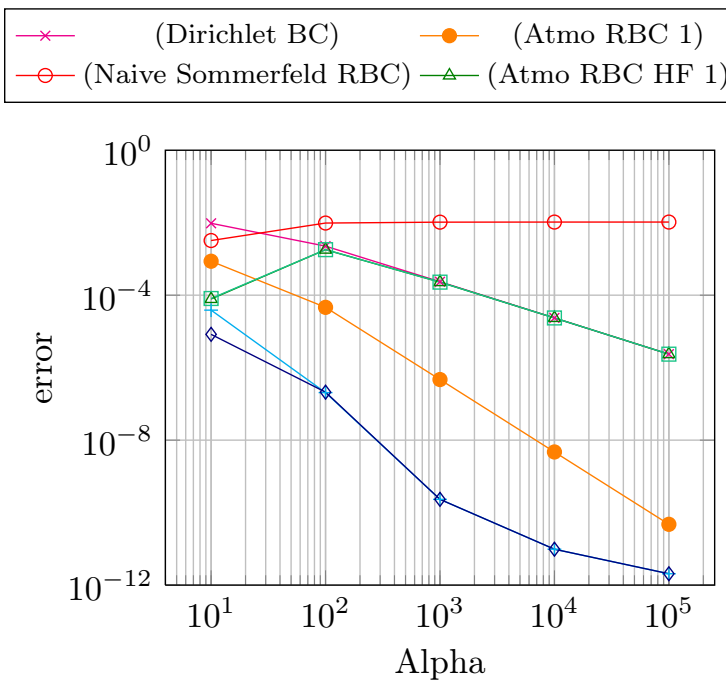

(a) Freq $=5.0 \mathrm{~Hz}$

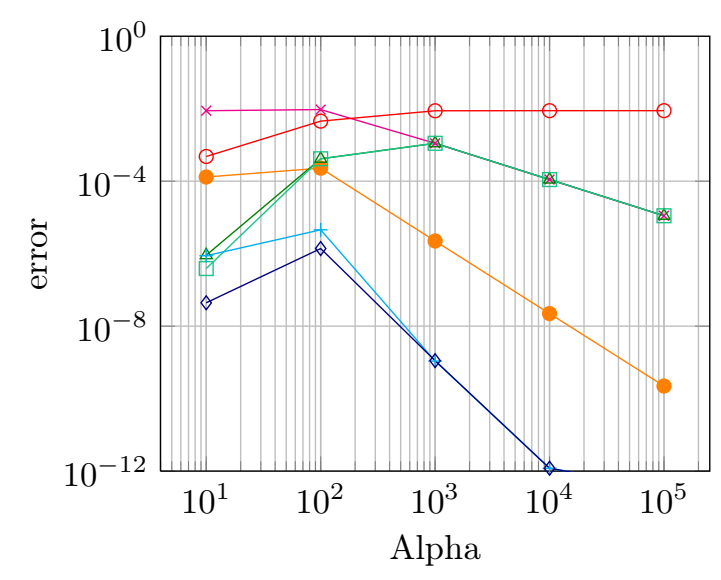

(b) Freq $=30 \mathrm{~Hz}$

Figure 4. $L^{2}$ relative errors with reference solution, with respect to the value $\alpha$.

The radius is set to $R=R_{a}$.

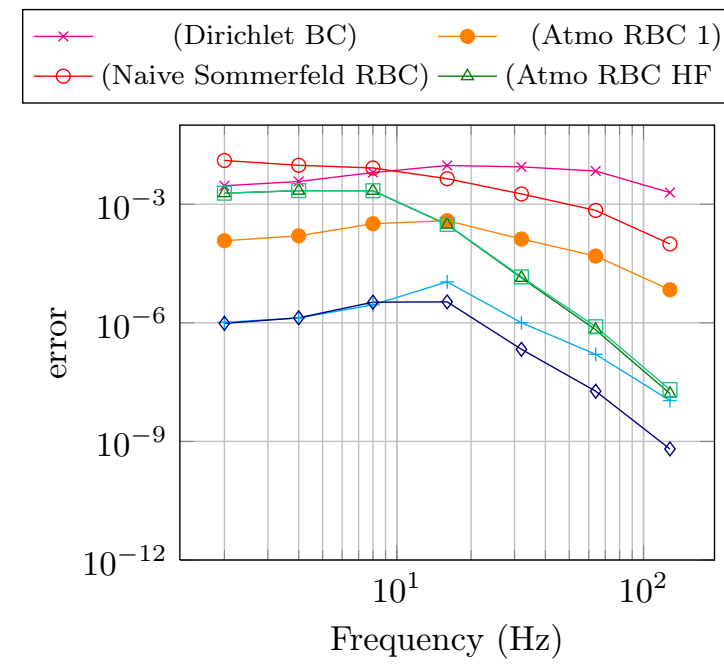

(a) $\alpha=50$ $\square($ Atmo RBC Non Spherical $) \multimap($ Atmo RBC Non Local $)$
$($ Atmo SAI 1$)$

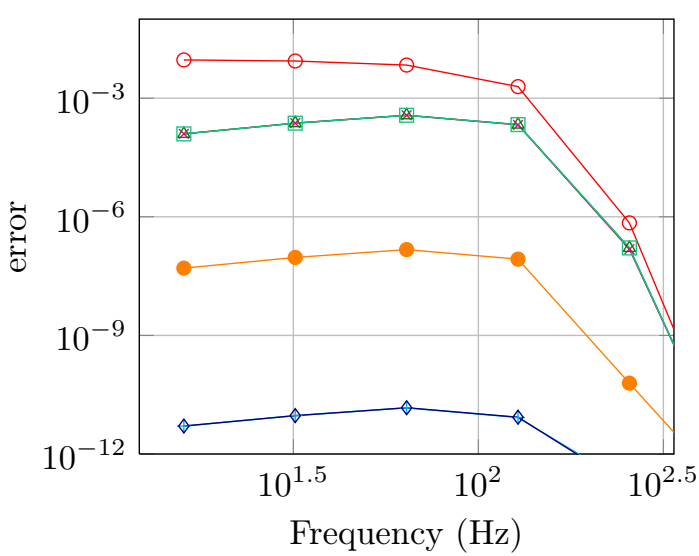

(b) $\alpha=5 \times 10^{3}$

Figure 5. $L^{2}$ relative errors with reference solution, with respect to the frequency. The radius is set to $R=R_{a}$.

\subsection{Sun atmosphere}

In the Sun, the coefficients are described by the Model S of [8]. In this paper, for accuracy reasons, we choose to use a background density $\rho$ and sound speed $c$ as regularized approximate values (with B-splines of order 8) of the Model S data. Fig. 6 displays the sound speed and density of the Model S inside the Sun. The atmospheric radius is considered to be equal to $R_{a}=1.000699 R_{\odot}$ where $R_{\odot}$ is the radius of the Solar surface. This corresponds to considering an idealized behavior after $500 \mathrm{~km}$ above the surface. The mesh size is chosen in order to have at least 10 degrees of freedom per wavelength, as explained in [9]. 


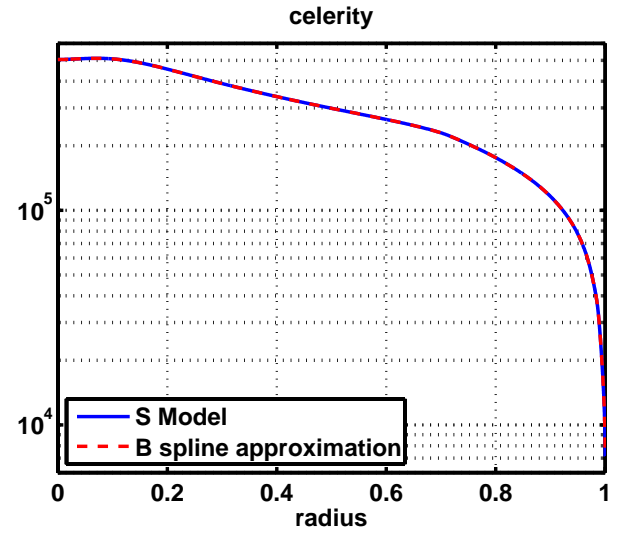

(a) Sound speed

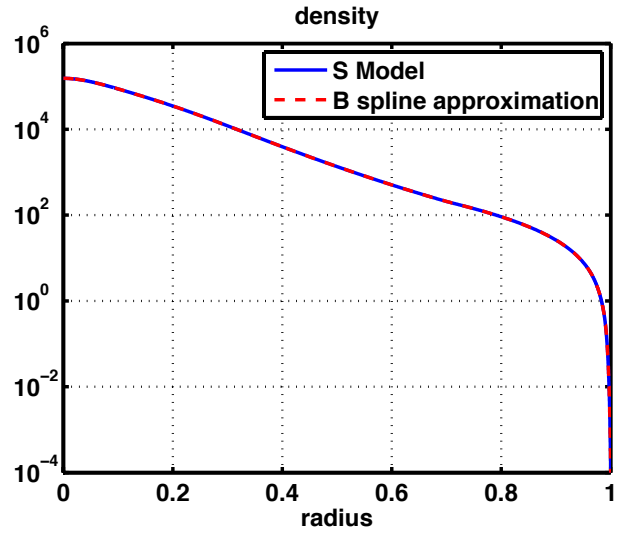

(b) Density

Figure 6. Sound speed (a) and Density (b) of Model S compared to the approximate value.

\subsubsection{Exponential decay in the atmosphere}

The parameter $\alpha$ is set to 7000. The damping parameter $\gamma$ defined in (3) is chosen as

$$
\gamma=\frac{\omega_{0}}{100} .
$$

The numerical experiments of this paragraph have been performed for a radial source, hence only the mode $\ell=0$ is involved. For this mode, the acoustic cut-off frequency is close to $5.3 \mathrm{mHz}$. The source is a Gaussian annulus located at $r \approx 0.99$ with a thickness equal to 0.008 .

We illustrate in Fig. 7 the fact that solutions are decaying exponentially in the atmosphere for three different frequencies. The figure also shows that the solution behaves differently depending on whether the frequency is below or above the acoustic cut-off frequency.

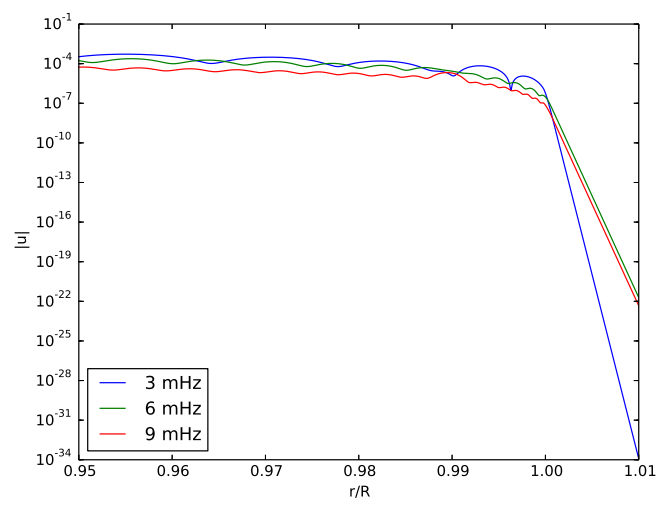

Figure 7 . Modulus of $u$ versus $r / R_{\odot}$ in logarithmic scale

\subsubsection{Influence of the distance of the artificial boundary}

In this section, we study the influence of the boundary condition and the value of $R$. The domain $[0, R]$ is meshed and the boundary condition is set on $r=R$. The source is a Gaussian located at $r \approx 0.99$ with a radius equal to 0.008 . We use $L=200$ modes for the reconstruction. The solution computed is compared with an exact solution obtained with the exact boundary condition 
discussed in Section 4.1. The atmosphere is present in the region $\left[R_{a}, R\right]$. We see in Table 1 that for $f_{0}=3 \mathrm{mHz}$, it is not necessary to mesh the atmosphere when using the atmospheric radiation boundary conditions (Atmo RBC 1) and (Atmo SAI 1), and that the solution becomes quickly independent from the boundary condition set on $r=R$.

\begin{tabular}{|c|c|c|c|}
\hline$R$ & $R_{a}$ & $1.0017 R_{a}$ & $1.003 R_{a}$ \\
\hline Absorbing & $5.647 \cdot 10^{-2}$ & $1.656 \cdot 10^{-4}$ & $8.222 \cdot 10^{-8}$ \\
Dirichlet & $1.723 \cdot 10^{-2}$ & $4.969 \cdot 10^{-5}$ & $2.467 \cdot 10^{-8}$ \\
(Atmo RBC 1) & $6.523 \cdot 10^{-6}$ & $1.862 \cdot 10^{-8}$ & $9.394 \cdot 10^{-12}$ \\
(Atmo SAI 1) & $6.061 \cdot 10^{-10}$ & $1.847 \cdot 10^{-12}$ & $1.594 \cdot 10^{-13}$ \\
\hline
\end{tabular}

TABLE 1. Relative $L^{2}$ error for different values of $R$ and boundary conditions for $f_{0}=3 \mathrm{mHz}$.

For higher frequencies (see Tables 2 and 3), we observe also that the boundary condition does not influence the solution if $R$ is large enough (here $R=1.2 R_{a}$ is sufficient), but a large region of the atmosphere must be meshed to achieve this result, except for conditions (Atmo RBC 1) and (Atmo SAI 1). These boundary condition can be imposed directly on the radius $R_{a}$ with a satisfactory result.

\begin{tabular}{|c|c|c|c|c|}
\hline$R$ & $R_{a}$ & $1.0017 R_{a}$ & $1.01 R_{a}$ & $1.2 R_{a}$ \\
\hline Absorbing & $2.625 \cdot 10^{-1}$ & $3.256 \cdot 10^{-1}$ & $1.494 \cdot 10^{-1}$ & $2.780 \cdot 10^{-9}$ \\
Dirichlet & $3.398 \cdot 10^{-1}$ & $6.514 \cdot 10^{-1}$ & $2.334 \cdot 10^{-1}$ & $4.267 \cdot 10^{-9}$ \\
(Atmo RBC 1) & $3.678 \cdot 10^{-4}$ & $3.349 \cdot 10^{-4}$ & $1.534 \cdot 10^{-4}$ & $2.810 \cdot 10^{-12}$ \\
(Atmo SAI 1) & $1.043 \cdot 10^{-7}$ & $9.480 \cdot 10^{-8}$ & $4.308 \cdot 10^{-8}$ & $2.692 \cdot 10^{-13}$ \\
\hline
\end{tabular}

TABLE 2. Relative $L^{2}$ error for different values of $R$ and boundary conditions for $f_{0}=6 \mathrm{mHz}$.

\begin{tabular}{|c|c|c|c|c|}
\hline$R$ & $R_{a}$ & $1.0017 R_{a}$ & $1.01 R_{a}$ & $1.2 R_{a}$ \\
\hline Absorbing & $2.766 \cdot 10^{-1}$ & $2.384 \cdot 10^{-1}$ & $1.357 \cdot 10^{-1}$ & $1.401 \cdot 10^{-7}$ \\
Dirichlet & $7.255 \cdot 10^{-1}$ & $7.385 \cdot 10^{-1}$ & $4.307 \cdot 10^{-1}$ & $4.127 \cdot 10^{-7}$ \\
(Atmo RBC 1) & $1.086 \cdot 10^{-4}$ & $1.009 \cdot 10^{-4}$ & $5.478 \cdot 10^{-5}$ & $4.739 \cdot 10^{-11}$ \\
(Atmo SAI 1) & $7.159 \cdot 10^{-9}$ & $6.644 \cdot 10^{-9}$ & $3.578 \cdot 10^{-9}$ & $8.370 \cdot 10^{-14}$ \\
\hline
\end{tabular}

TABLE 3. Relative $L^{2}$ error for different values of $R$ and boundary conditions for $f_{0}=9 \mathrm{mHz}$.

Finally in Fig. 8 the $L^{2}$ relative error for the same case is displayed with respect to the frequency for four different atmosphere sizes. If one wishes to ensure a given precision, for instance $10^{-5}$, for all frequencies of a given range, for instance $[3.0,12.0] \mathrm{mHz}$, as in helioseismology, these curves show the necessary size of meshed atmosphere for each boundary condition. When using Dirichlet 
boundary condition, the meshed atmosphere size must be $1.2 R$. When using (Atmo RBC 1 ), $1.05 R$ is sufficient. When using (Atmo SAI 1), the condition can be put directly on the surface, the accuracy will be better than $10^{-5}$ for all frequencies. The computational burden is therefore diminished drastically in terms of memory and CPU-time thanks to this new condition since the mesh can be significantly smaller for the same accuracy (see [12] for quantitative comparisons on real data).

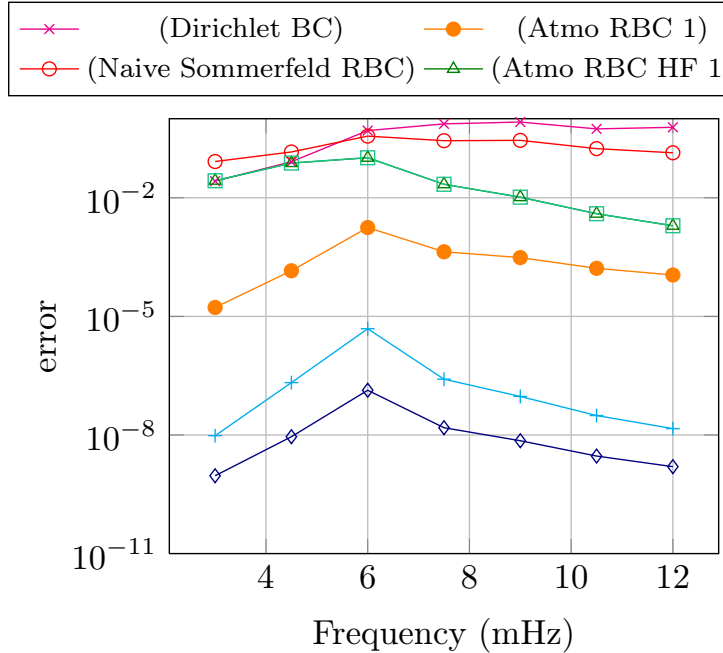

(a) OSRC

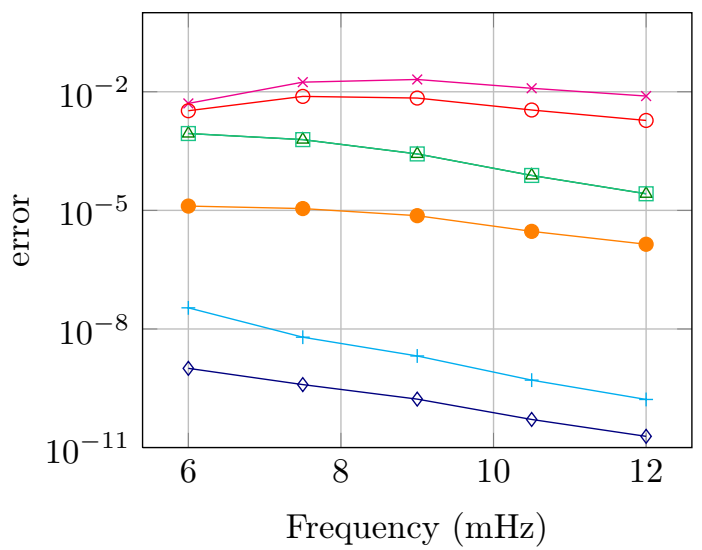

(c) $1.05 R$

\section{(Atmo RBC Non Spherical $) \multimap($ Atmo RBC Non Local $)$ (Atmo SAI 1)}

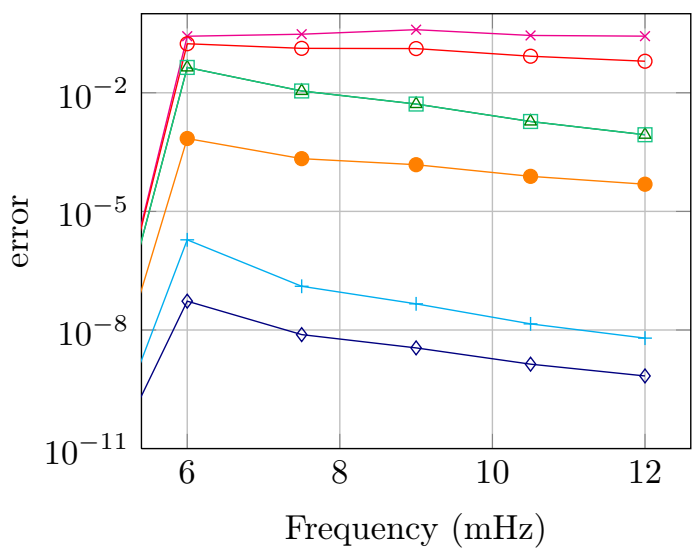

(b) $1.01 R$

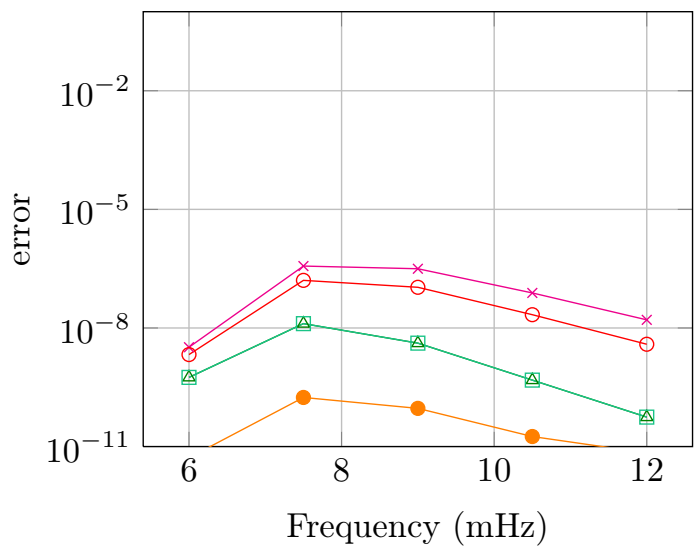

(d) $1.2 R$

FiguRE 8. L2 relative errors with reference solution, with respect to the frequency. Volumic source with 200 modes.

\subsubsection{Power spectra}

In this paragraph, we show the influence of the $\mathrm{BC}$ in the helioseismology context, by computing oscillation power spectra with different BCs. The power spectrum measures the amplitudes of the $\ell$-modes depending on the frequency $\omega_{0}$. The oscillation power spectrum contains most of the information needed for the interpretation of helioseismic observations.

Following [9], we consider a radial setup, in which we compute the Green's functions $G^{\ell, m}(\omega)$ by solving Eq. (1) where the source $f$ is a Dirac point source. This source is located at the Solar 


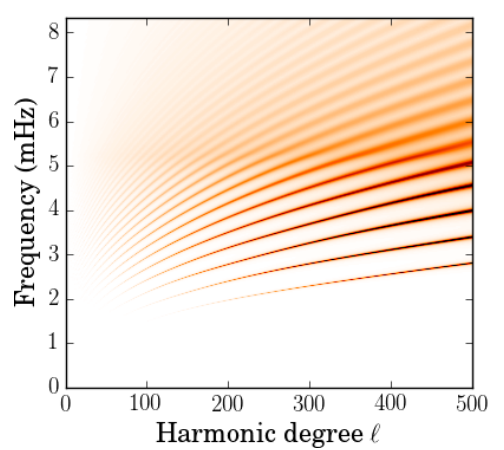

(a) Reference solution with meshed atmosphere

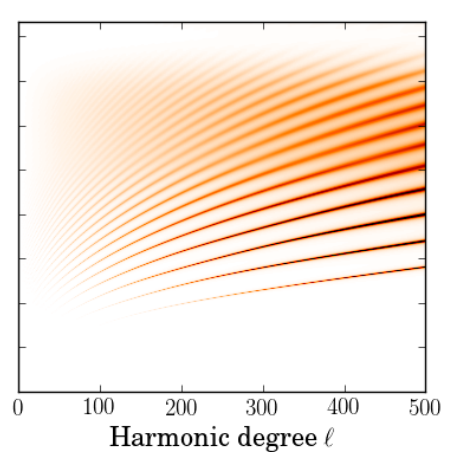

(b) Dirichlet BC

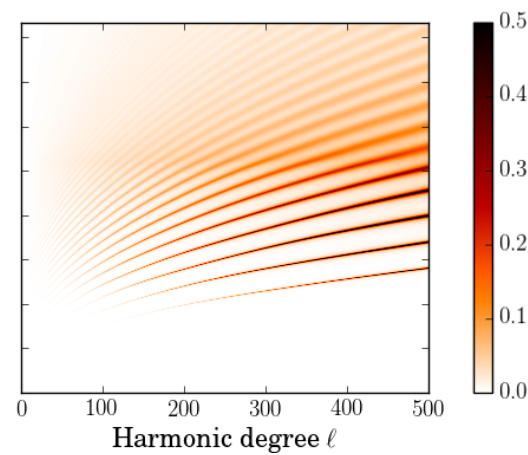

(c) (Atmo RBC Non Local)

Figure 9. Power spectra obtained with Model S data.

surface $\left(r=R_{\odot}<R_{a}\right)$, at a point such that the only non-zero contributions of Eq. (7) are obtained for $m=0$. Therefore, we solve for each harmonic degree $\ell$ and each frequency:

$$
-\frac{\omega^{2}}{c^{2}} G^{\ell, 0}(\omega)-\frac{1}{r^{2}} \frac{\partial}{\partial r}\left(r^{2} \frac{\partial G^{\ell, 0}(\omega)}{\partial r}\right)+\frac{\ell(\ell+1)}{r^{2}} G^{\ell, 0}(\omega)-\alpha \frac{\partial G^{\ell, 0}}{\partial r}=\rho \delta_{R_{\odot}}(r) .
$$

Adapting the definition given in [9], the oscillation power spectrum is directly given by

$$
\mathcal{P}^{\ell}(\omega)=\operatorname{Im}\left(G^{\ell, 0}\left(R_{\odot}, \omega\right)\right) / \omega .
$$

Simulation parameters were set as in [9], i.e.:

- Density and sound speed data are taken from Model S [8] and regularized using $8^{\text {th }}$-order B-splines (Fig. (6)). The parameter $\alpha$ is deduced from the last values of Model $\mathrm{S}$ and set to $\alpha \sim 6633$.

- The damping parameter $\gamma$ was set to a power law in order to fit solar data:

$$
\gamma(\omega)=\gamma_{0}\left|\frac{\omega}{\omega_{1}}\right|^{\beta},
$$

with $\gamma_{0} / 2 \pi=8.58 \mu \mathrm{Hz}, \omega_{1} / 2 \pi=3.00 \mathrm{mHz}$ and $\beta=5.77$.

- Green's functions were computed for 8000 frequencies ranging from 0 to $8.3 \mathrm{mHz}$.

The computational mesh is a sampling of the Model $\mathrm{S}$ data, which is more refined close to the surface. When needed, the mesh is extended in the atmosphere with a regular grid that matches Model S resolution at $r=R_{a}$.

As a reference, the power spectrum is computed in an extended domain with a Dirichlet BC set at $R=1.05 R_{\odot}=1.0493 R_{a}$. The value $R$ corresponds to the height at which waves are completely attenuated before reaching the boundary. Power spectra are then computed with both Dirichlet and (Atmo RBC Non Local) conditions at $R=R_{a}$. Fig. 9 shows good agreement between the reference Power Spectrum and the computation with (Atmo RBC Non Local) condition. In particular, amplitudes of high frequencies modes are well captured, contrary to the Dirichlet BC.

Using the newly proposed RBC then represents a major computational gain, as computing all Green's functions is performed using 2.69 CPU-hours instead of 4.71 CPU-hours with the extended atmosphere. 

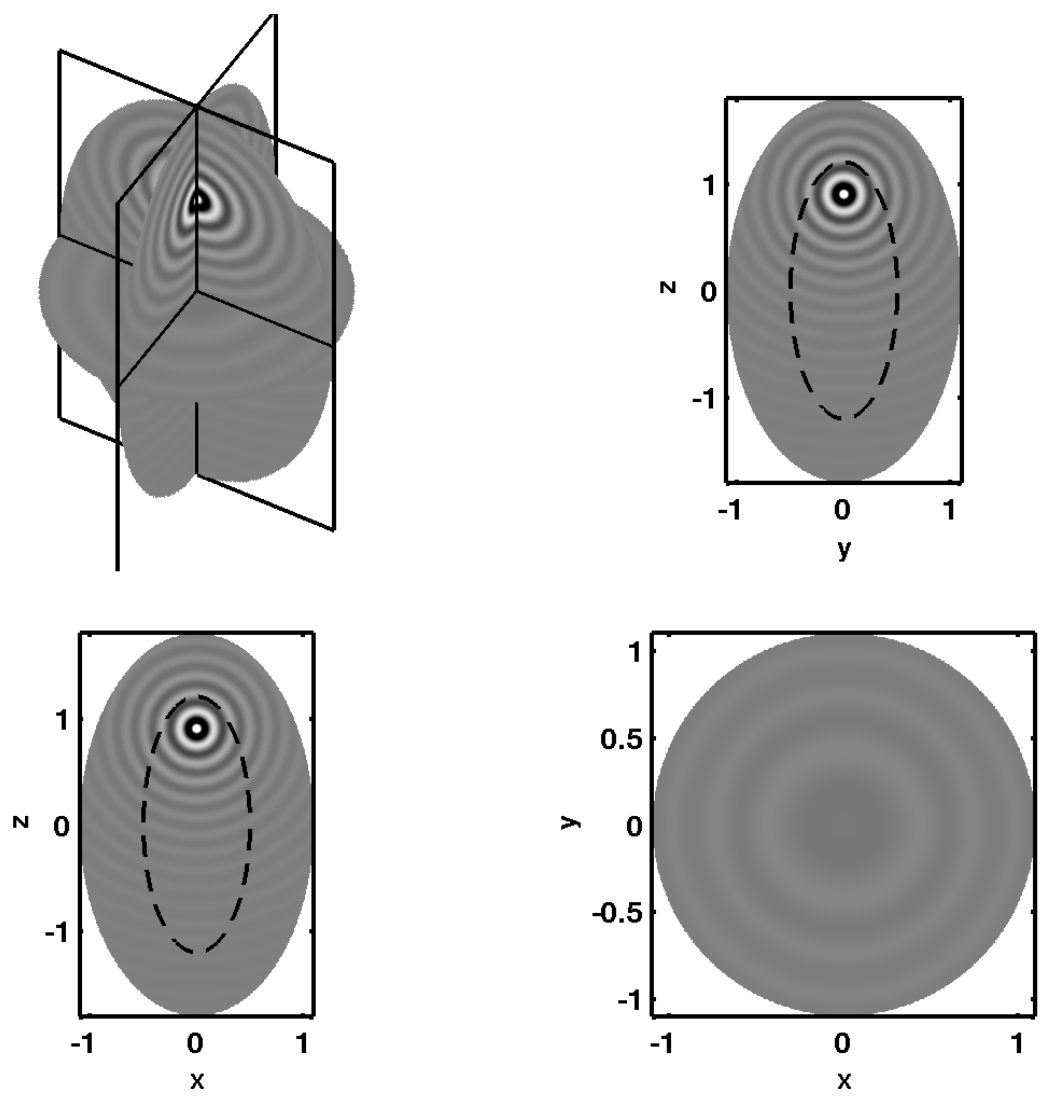

Figure 10. Real part of the reference solution for the ellipse. The dashed line represents the ellipse.

\subsection{Non spherical geometry}

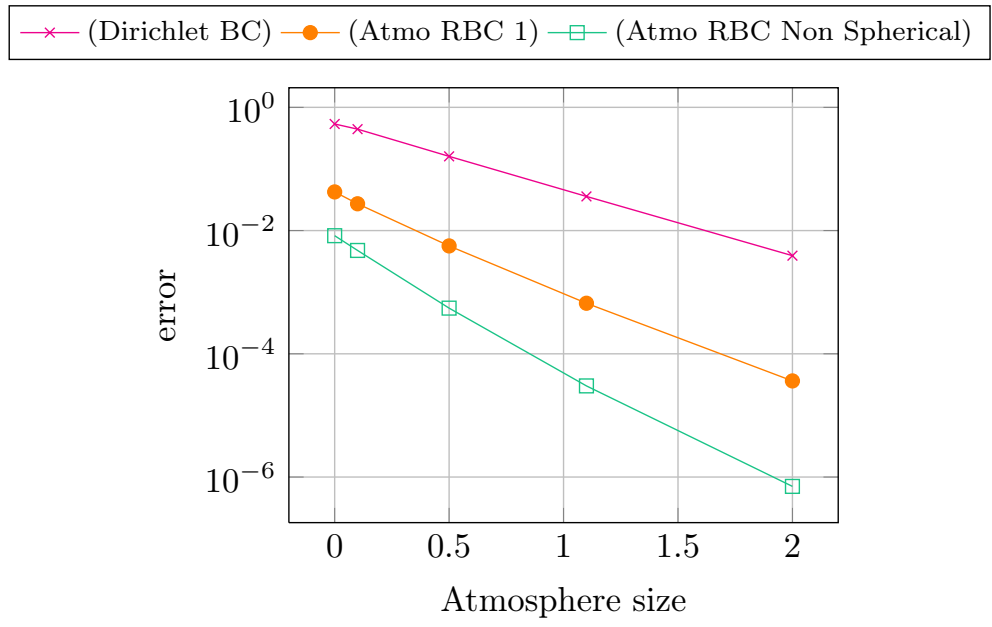

FiguRE 11. $L^{2}$ relative errors with reference solution, with respect to the size of meshed atmosphere. Axisymmetric computation. 
In this paragraph, we assess the performance of the boundary conditions set on a non spherical geometry: an ellipse. In this case, the radial FEM setting described above is not suited anymore, so the computations are done using an axisymmetric setting, see [6]. We compare the use of Dirichlet boundary conditions with (20) which has been especially derived for non radial geometries, and (Atmo RBC 1) which comes from the factorization of the Helmholtz equation in a radial context. The medium is defined as follows:

$$
\rho(x, y)=\left\{\begin{array}{ll}
2.0 & \text { if }\left(\frac{x}{a}\right)^{2}+\left(\frac{y}{b}\right)^{2} \leq 1, \\
2.0 e^{-\alpha d(x, y)} & \text { if }\left(\frac{x}{a}\right)^{2}+\left(\frac{y}{b}\right)^{2}>1,
\end{array} \quad c(x, y)=1.0,\right.
$$

where $d(x, y)$ is the distance from the point $(x, y)$ to the ellipse. The parameter $\alpha$ is set to 1.0 and the ellipse parameters are $a=0.5$ and $b=1.2$. The damping parameter is $\gamma=6.0$, and the frequency is chosen as $f_{0}=6 \mathrm{~Hz}$. The source is a Gaussian located at the point $(0,0,0.9)$ with a radius of 0.05 . The domain is arbitrarily truncated by the elliptic interface defined by characteristic lengthes $\tilde{a}$ and $\tilde{b}$ larger than $a$ and $b(\tilde{b}=C \times b, C>1)$ and such that $\tilde{a}-a=\tilde{b}-b$. In Fig. 11 the relative $L^{2}$ error on the vertical line $[(0,0,-b),(0,0, b)]$ is displayed with respect to the relative atmosphere size. The reference solution for each boundary condition is obtained for an atmosphere size ratio of 5.0, using the same boundary condition. This reference solution is displayed in Fig. 10, for condition (Atmo SAI 1). It is clear that in this case, condition (20) outperforms the other ones. Especially, when the condition is set directly on the boundary of the ellipse, the relative $L^{2}$ error is less than $1 \%$, while imposing a Dirichlet boundary condition leads to an error larger than $50 \%$.

\section{Conclusions And PROspects}

This work focused on the computation of acoustic waves propagating in the Sun and its atmosphere, which, in this paper, is defined as the idealized region where the sound speed is constant and the density decays exponentially with radius. We have derived a collection of non-reflecting boundary conditions to model waves that propagate away from a given surface used to truncate the idealized part of the Sun atmosphere. A first factorization of the problem in spherical coordinates has led to a characterization of outgoing waves and to a non-local radiation condition. The associated problem is well posed but is computationally expensive due to its nonlocal nature in 3D. Approximate radiation boundary conditions have therefore been proposed for high frequencies or small angles of incidence. Finally, a radiation boundary condition has been proposed for high frequencies for non spherical geometries. All these conditions have been implemented with finite elements in radial and axisymmetric configurations along with the computation of a reference solution. Systematic tests have been performed in a toy model, where we have studied the influence of many factors (parameter of the exponential decay, distance of the boundary, frequency). When usable, the nonlocal condition (Atmo RBC Non Local) outperforms the other ones in terms of precision, closely followed by condition (Atmo SAI 1) which turns out to be the best option in axisymmetric configurations. The derived conditions perform also very well in the realistic configuration of the Sun, for all frequencies of interest, leading to a drastic decrease of the computational burden since meshing the atmosphere is not necessary anymore. The computation of oscillation power spectra show that (Atmo RBC Non Local) captures well the behavior of the waves especially at high frequency, while diminishing the numerical burden by a factor two. This last result is a significant contribution to helioseismology.

This prompts us to the next step of this work, which is motivated by probing the Sun interior via inversion methods. In this context, the exponential decay coefficient could be considered as another unknown of this inversion process. However, depending on the condition, this can be a difficult task due to the nonlinear dependency of the conditions with respect to this coefficient. Another interesting topic that we aim at considering is to propose and analyze atmospheric radiation boundary conditions in the time domain. 


\section{ACKNOWLEDGEMENTS}

This project has received funding from the European Union's Horizon 2020 research and innovation program under the Marie Sklodowska-Curie grant agreement No 644602. This project has received funding from the Agence Nationale de la Recherche under the grant NumForSun No ANR-16-MRS3-0019-01.

\section{REFERENCES}

[1] X. Antoine, Fast Approximate Computation of a Time-Harmonic Scattered Field using the On-Surface Radiation Condition Method. IMA Journal of Applied Mathematics, vol 66, pp 83-110, 2001.

[2] X. Antoine, H. Barucq and A. Bendali, Bayliss-Turkel-like radiation conditions on surfaces of arbitrary shape. Journal of Mathematical Analysis and Applications, vol 229, pp 184-211, 1999.

[3] H. Barucq, R. Djellouli, A. G. Saint-Guirons, Performance assessment of a new class of local absorbing boundary conditions for elliptical- and prolate spheroidal-shaped boundaries. Applied Numerical Mathematics, Elsevier, vol 59(7), pp 1467-1498, 2009.

[4] H. Barucq, R. Djellouli, A. G. Saint-Guirons, Three-dimensional approximate local DtN boundary conditions for prolate spheroid boundaries. Journal of Computational and Applied Mathematics, Elsevier, vol 234(6), pp 1810-1816, 2010.

[5] A. Bayliss, M. Gunzburger, and E. Turkel, Boundary conditions for the numerical solution of elliptic equations in exterior regions. SIAM Journal of Applied Mathematics, vol 42, pp 430-451, 1982.

[6] J. Chabassier, M. Duruflé, High Order Finite Element Method for solving Convected Helmholtz equation in radial and axisymmetric domains. Application to Helioseismology. Inria Bordeaux Sud-Ouest Research Report, RR-8893, pp 1-54, 2016.

[7] J. Chabassier, M. Duruflé and V. Péron, Equivalent boundary conditions for acoustic media with exponential densities. Application to the atmosphere in helioseismology. Inria Bordeaux Sud-Ouest Research Report, RR8954, pp 1-32, 2016.

[8] J. Christensen-Dalsgaard et al. The current state of solar modeling. Science, vol 272, pp 1286 - 1292, 1996.

[9] L. Gizon, H. Barucq, et al. Computational helioseismology in the frequency domain: acoustic waves in axisymmetric solar models with flows. Astronomy \& Astrophysics, vol. 600, A35, 2017.

[10] A. G. Kosovichev, Advances in Global and Local Helioseismology: An Introductory Preview. The Pulsations of the Sun and the Stars, Lecture Notes in Physics, Springer, vol. 832, pp 3-84, 2011.

[11] L. Gizon, A.C. Birch and H.C. Spruit, Local Helioseismology : Three-dimensional imaging of the Solar interior. Annual Review of Astronomy and Astrophysics, vol. 48, pp 289-338, 2010.

[12] D. Fournier, M. Leguèbe, et al. Atmospheric radiation boundary conditions for high frequency waves in time-distance helioseismology. submitted to Astronomy and Astrophysics, 2017.

[13] L. Nirenberg, Lectures on Linear Partial Differential Equations, CBMS Regional Conference Series in Mathematics No 17, American Mathematical Society, Providence, RI, 1973. MR0450755 (56:9048)

[14] S. H. Schot, Eighty Years of Sommerfeld's Radiation Condition. Historia Mathematica, 19, pp 385-401, 1992.

[15] H. Schunker, R. H. Cameron, L. Gizon, H. Moradi, Constructing and Characterising Solar Structure Models for Computational Helioseismology. Solar Physics, vol 271, pp 1-26, 2011. 